Research Paper

\title{
Homer2 and Homer3 Act as Novel Biomarkers in Diagnosis of hepatitis B virus-induced Hepatocellular Carcinoma
}

\author{
Ping Luo ${ }^{1,6^{*}}$, Chunzi Liang6* $6^{*}$ Wei Jing'2, Man Zhu ${ }^{3}$, Hu Zhou ${ }^{4}$, Hongyan Chai ${ }^{6}$, Paul F. Worley5 , Jiancheng \\ $\mathrm{Tu}^{6}$ \\ 1. Department of Hematology, Zhongnan Hospital of Wuhan University, Wuhan, China \\ 2. Department of Clinical Laboratory, Key Laboratory of Laboratory Medicine of Henan, the First Affiliated Hospital of Zhengzhou University, Zhengzhou \\ 450000, China. \\ 3. Department of Medical Laboratory, The Central Hospital of Wuhan, Tongji Medical College, Huazhong University of Science and Technology, Wuhan, \\ Hubei 430014, P.R. China. \\ 4. Department of Transfusion, Tongii Hospital, Tongji Medical College, Huazhong University of Science and Technology. \\ 5. Department of Neuroscience, School of Medicine, The Johns Hopkins University, Baltimore, MD 20205, USA. \\ 6. Department \& Program of Clinical Laboratory Medicine, Center for Gene Diagnosis, Zhongnan Hospital of Wuhan University, Wuhan 430071, China. \\ * These authors contributed equally \\ $\triangle$ Corresponding author: Jiancheng Tu, E-mail: jianchengtu@whu.edu.cn
}

(c) The author(s). This is an open access article distributed under the terms of the Creative Commons Attribution License (https://creativecommons.org/licenses/by/4.0/). See http:/ /ivyspring.com/terms for full terms and conditions.

Received: 2020.08.18; Accepted: 2021.03.20; Published: 2021.04.19

\begin{abstract}
Background: Hepatocellular carcinoma ( $\mathrm{HCC})$ is one of the most common causes of cancer-related mortality worldwide. Early detection of $\mathrm{HCC}$ can significantly improve patients' outcomes. An increasing number of studies have validated that Homer is dysregulated in cancers and may serve as diagnostic markers. In the present study, we investigated the expression profile and diagnostic significance of Homer2 and Homer3 in hepatitis B virus-induced HCC (HBV-HCC).

Methods: Quantitative real-time PCR (QRT-PCR), western blot analysis and immunohistochemistry analysis.

Results: Homer2 and Homer3 were downregulated in HCC. The expression of Homer2 was associated with tumor differentiation grade $(P=0.012)$ and total protein (TP) level $(P=0.032)$. Homer3 was related to tumor size $(P=0.010)$, tumor nodes $(P=0.026)$ and $y$-glutamyl transferase (GGT) level $(P=0.001)$. The receiver operating characteristic curve analyses indicated that the combination of Homer2, Homer3 and AFP possessed a high accuracy ( $\mathrm{AUC}=0.900$ ) to diagnose $\mathrm{HCC}$ cases from healthy controls.

Conclusion: Our data indicated that Homer2 and Homer3 were downregulated in $\mathrm{HCC}$ and might be potential diagnostic marker for HCC.
\end{abstract}

Key words: Homer2; Homer3; HCC; Tumorigenesis; Diagnosis.

\section{Introduction}

The mortality of Liver cancer ranks second among human cancers worldwide [1, 2]. It is reported that hepatocellular carcinoma (HCC) occupy approximately $80 \%$ of liver cancers [3]. In 2018, about 840,000 HCC patients were newly diagnosed and 782,451 deaths were reported. Furthermore, it is estimated that by 2030, the new cases of HCC may reach to 1 million [4]. Among many reported risk factors of HCC, cirrhosis caused by chronic hepatitis B or $C$ virus infections is the major one [5-7]. Data has shown that the 5-year-survival rate of HCC patients remained less than $12 \%$ due to the delayed diagnosis [8]. Though early diagnosis, together with interventional therapy and/or surgical methods can significantly improve the outcome of HCC, unfortunately the majority of HCC patients are 
diagnosed at an advanced stage [3]. Currently, serum alpha-fetoprotein (AFP) levels combined with computed tomography (CT) and magnetic resonance imaging (MRI) are the most common non-invasive diagnostic methods, but all of them have sensitivity problems [9]. Hence, new non-invasive biomarkers with high sensitivity and specificity for the early diagnosis of HCC, especially among high-risk people such as patients with the chronic liver disease would be highly beneficial.

Homer protein families in mammals are mainly made up of three members, Homer1, Homer2 and Homer3, each of them has several isoforms due to alternative splicing $[10,11]$. It is reported that all Homer family members have a common EVH1 domain at the N-terminus [12]. In 2008, Derek and his colleagues found that Homer3 was over expressed in Acute Myelocytic Leukemia (AML) samples with favorable cytogenetics [13]. In 2016, Shen et al. revealed that Homer3 were overexpressed in esophageal squamous cell carcinoma, and significantly linked with pN and pStage [14]. In 2017, Sun et al. [15] revealed that the expression of Homer2 was increased in rectal carcinoma (RC) via co-expression network analysis. They also found that Homer2 expression was correlated with higher pathological stage and shorter overall survival of RC patients. Recently, another study concluded that lowgrade endometrioid endometrial adenocarcinoma patients with high expression of Homer2 protein had a better outcome [16]. Thus, we hypothesize that Homer plays an important role in the process of tumors. Our previous research has revealed that Homer1, downregulated both in the HCC cell line \& tissues, can be used as a diagnostic maker for HBV-HCC [17]. Toward this direction, we assumed an association between Homer2, Homer3 and HCC. Huang et al. [18] found that Homer2 and Homer3 can negatively regulate the activation of $\mathrm{T}$ cell through competing with calcineurin and by binding of nuclear factor of activated $\mathrm{T}$ cells (NFAT). T cell associated immune response were related to the survival of HCC [19], which further supported our hypothesis.

Here, we aim to evaluate the expression level of Homer2 and Homer3 \& its correlation with clinicopathological characteristics of patients with HBV-HCC, and then to explore the value of novel biomarker of Homer2 and Homer3 in the diagnosis of HBV-HCC.

\section{Materials and Methods}

\section{Sample collection}

A total of 77 HCC (71 men and 6 women, mean age $58 \pm 10)$ tissues and paired adjacent non-tumor tissues were collected from HCC patients who underwent surgical resections from October 2014 and October 2019 at Zhongnan Hospital of Wuhan University. At the same time, 183 patients' whole blood samples from the same hospital during 2016 were collected. Patients were classified into three group: 72 patients with HCC (59 males and 13 females, mean age $57 \pm 13$ ), 52 patients with hepatitis B ( 37 males and 15 females, mean age $52 \pm 14$ ), and 59 the cirrhosis (49 males and 10 females, mean age 56 \pm 12 ), Meantime, 109 healthy blood samples (80 males and 29 females, mean age 54 \pm 11 ) were collected from the Physical Examination Center. All of the patients were selected based on medical or pathology reports. The inclusion criteria were as follows: HBsAg positive and without any other liver diseases, such as alcoholic, autoimmune, hepatitis $\mathrm{C}$ and metabolic liver diseases; newly diagnostic. Exclusion criteria were as follows: patients with history of chemotherapy or radiotherapy; patients with no comprehensive clinical data. The species were stored at $-80^{\circ} \mathrm{C}$ until RNA extraction.

\section{RNA extraction and cDNA synthesis}

Total RNA was extracted using Trizol reagent (Invitrogen, Carlsbad, CA, USA) as described by the manufacturer. After extraction, RNA concentration was measured using Nanodrop 2000 spectrophotometer (Thermo Scientific Inc., Waltham, MA, USA). The reverse transcription (RT) reaction of $1 \mu \mathrm{g}$ RNA was performed with random primers in a final volume of $20 \mu \mathrm{L}$ using PrimeScriptTM RT reagent Kit with gDNA Eraser (Takara, Japan). Reverse transcription conditions were as follows: $42^{\circ} \mathrm{C}$ for $2 \mathrm{~min}$, and then $37^{\circ} \mathrm{C}$ for $15 \mathrm{~min}, 85^{\circ} \mathrm{C}$ for $5 \mathrm{~s}$, followed by storage at $4^{\circ} \mathrm{C}$. All cDNA samples were stored at $-80^{\circ} \mathrm{C}$ before real-time PCR analysis.

\section{Real-Time Polymerase Chain Reaction}

Real-Time PCR was performed on Bio-Rad CFX96 (Bio-Rad Laboratories, Inc., Hercules, CA, USA) using SYBR-Green I Premix Ex Taq following manufacturer's instructions. The reaction was carried out in a volume of $20 \mu$ l containing $1.5 \mu \mathrm{L}$ cDNA, $10 \mu \mathrm{L}$ SYBR Green mix, $1.6 \mu \mathrm{L}$ gene-specific forward and reverse primers $(10 \mu \mathrm{M})$, and $6.9 \mu \mathrm{L}$ nuclease-free water. Glyceraldehyde-3-phosphate dehydrogenase (GAPDH) was selected as housekeeping gene for normalization. The primers for the reaction were as follows: Homer2 sense: 5'-TCACCGTTTCCTACT TCTATG-3' and antisense: 5'-CCTGCGTCTTGT CTT-TGG-3'; Homer3 sense: 5'-CGCACTCACTGT CTCCTATT-3' and antisense: 5'-GGAACTTCTCGG -CAAACT-3'; GAPDH sense: 5'-AGAAGGCTGGGG CTCATTTG-3' and antisense: 5'-GCAGGAGGCATT 
GCTGATGAT-3'. The amplifications started at $95^{\circ} \mathrm{C}$ for $5 \mathrm{~min}$ followed by 40 cycles, each consisting of denaturing for $30 \mathrm{~s}$ at $95^{\circ} \mathrm{C}$, annealing for $30 \mathrm{~s}$ at $62^{\circ} \mathrm{C}$, and elongation for $30 \mathrm{~s}$ at $72^{\circ} \mathrm{C}$. All reactions were amplified in duplicate with no-template controls included. Amplification specificity for each gene was confirmed by a single distinct melting curve. PCR products were separated using $2.0 \%$ agarose gel electrophoresis to confirm the presence of a single band at the expected amplified size.

\section{Cells and cell culture}

The normal human hepatocyte cell line L-02 were bought from the Procell Inc. (Wuhan, China). HCCLM-9 were maintained at our laboratory. We purchased human HCC cell lines HepG2 from the China Center for Type Culture Collection (CCTCC, Wuhan, China). All the three Cells were cultured in RPMI 1640 medium (Gibco, USA) containing 10\% fetal bovine serum (FBS, Gibco, USA), $50 \mathrm{U} / \mathrm{ml}$ penicillin and $50 \mathrm{U} / \mathrm{ml}$ streptomycin.

\section{Western blot analysis}

The stable cells were lysed using RIPA lysis buffer in the presence of PMSF proteinase inhibitor (Beyotime, China). Approximately a total of $40 \mathrm{ug}$ protein was electrophoresed on $12 \%$ sodium dodecyl sulphate polyacrylamide gel and transferred onto polyvinylidene difluoride (PVDF) membrane. The membrane was blocked with 5\% non-fat milk in PBST before incubation with the primary antibody. The primary antibodies used for Homer2 and Homer3 were mouse polyclonal (Abcam, USA). GAPDH was used as a loading control. After incubated with secondary antibody for $1 \mathrm{~h}$ at room temperature, the signals were visualized with enhanced chemiluminescence (ECL) (Beyotime, China).

\section{Immunohistochemistry analysis}

Cells were seeded in 6-well plates, fixed in methanol for $15 \mathrm{~min}$, and then permeabilized with $0.2 \%$ Triton X-100 for $15 \mathrm{~min}$. Endogenous peroxidase activity was blocked by incubating in $3 \% \mathrm{H}_{2} \mathrm{O}_{2}$ solution. Next, the cells were blocked with goat serum for 30min and incubated with Homer2 and Homer3 antibodies (1:100, Abcam, USA) overnight at $4{ }^{\circ} \mathrm{C}$. Subsequently, samples were incubated with the corresponding HRP-conjugated secondary antibody solution for $45 \mathrm{~min}$. And then DAB (DAB-0031; Fuzhou, China) substrate solution was applied for antibody staining for $10 \mathrm{~min}$. Nuclei were stained with hematoxylin.

\section{Statistical Analysis}

We presented normally distributed data as mean \pm standard deviation (SD) and skewed data as median and inter-quartile range. The Shapiro-Wilk test was carried out to check the normality of the distribution. For normally distributed data, student's $t$ test was used to compare 2 groups of continuous variables and one-way ANOVA was used for the comparison among multiple groups, while non-normally distributed data was analyzed by Kruskal-Wallis variance analysis. Qualitative data was tested using Chi-square test. To evaluate the diagnostic value of Homer2 and Homer3, receiver operating characteristic (ROC) curves were made, and the area under the ROC curves was determined. The statistical analyses were performed with the SPSS 17.0 software (SPSS, Chicago, IL, USA) and GraphPad Prism 5 software (GraphPad software, La Jolla, CA, USA). All statistical tests were two-sided and $P<0.05$ was considered statistically significant.

\section{Results}

\section{Homer2 and Homer3 expression in HCC cell lines}

QRT-PCR, Western blot analysis and immunohistochemical method were used to detect the expression of Homer2 and Homer3 in several cell lines (L-02, Hep-G2, and HCCLM-9). We found that both Homer2 and Homer3 expression were significantly higher in L-02 cell line than in other cell lines (Hep-G2 and HCCLM-9) in RNA and protein level (Figure 1).

\section{Homer2 and Homer3 were down-regulated in HCC tissues}

RT-qPCR was used to detect the expression levels of Homer2 and Homer3 in HCC tissues and paired normal liver tissues derived from 77 HCC patients. Our results revealed that the relative expression levels of Homer2 and Homer3 were down-regulated in HCC tissues compared with matched adjacent tissues. (Homer2: $P<0.01$, Figure 2A; Homer3: $P<0.01$, Figure 2B). Then, waterfall plot demonstrated that Homer2 was reduced by at least twofold in 53.2\% (41/77) of the HCC tissues (Figure 2C). We also found that Homer3 was downregulatedin $62.3 \%(48 / 77)$ of HCC tissues (Figure 2D).

\section{Clinicopathological data analysis of HCC patients}

The detailed clinic parameters of enrolled 77 HCC tissue patients and clinicopathological relevance analysis were summarized in Table $\mathbf{1}$. Of all the 77 patients, 71 are male and 38 patients are over the age of 55. As to the severity of the tumor, 9 patients are low differentiation, 47 patients with tumor size bigger than $5 \mathrm{~cm}, 29$ patients belongs to TNM III IV and 8 patients had multi tumor nodes. The number of 
patients with AFP, GGT higher than normal value is 32,42 and the numer of patients with TP and Alb lower than normal value are 22, 12. Correlation analysis results showed that Homer2 expression was significantly associated with tumor differentiation grade $(P=0.012)$ and $\mathrm{TP}(P=0.032)$, while the expression of Homer3 was significantly related to tumor size $(P=0.010)$, tumor nodes $(P=0.026)$ and GGT level $(P=0.001)$. Thus, we hypothesized that down-regulation of Homer2 and Homer3 might play

$\mathbf{A}$

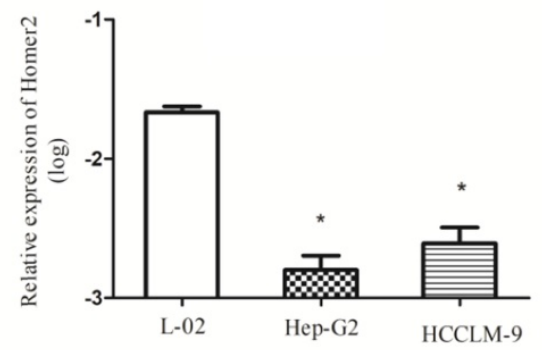

C

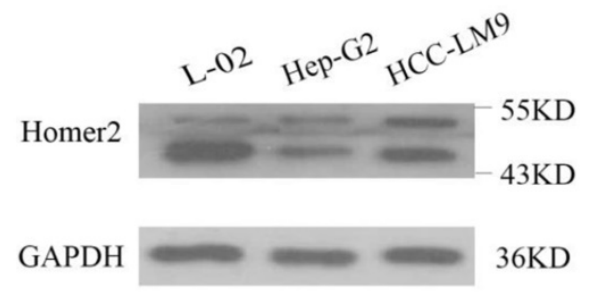

D
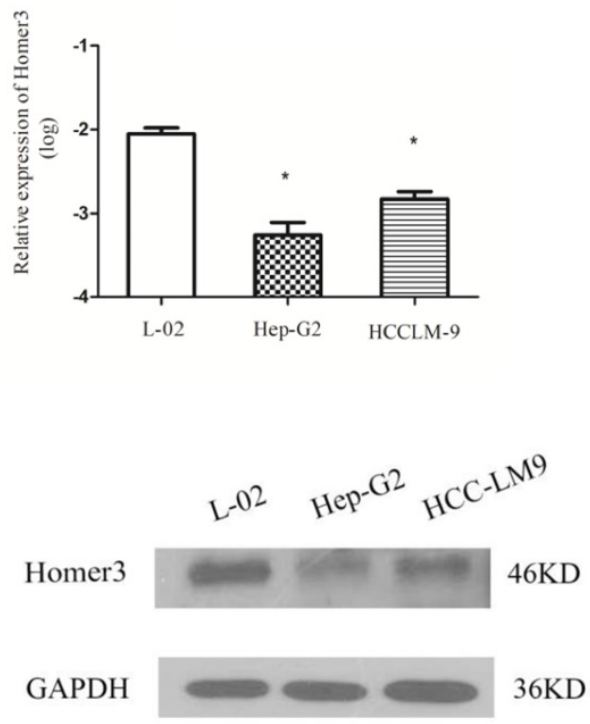

$\mathbf{E}$ Homer2

\section{$\mathbf{F}$}

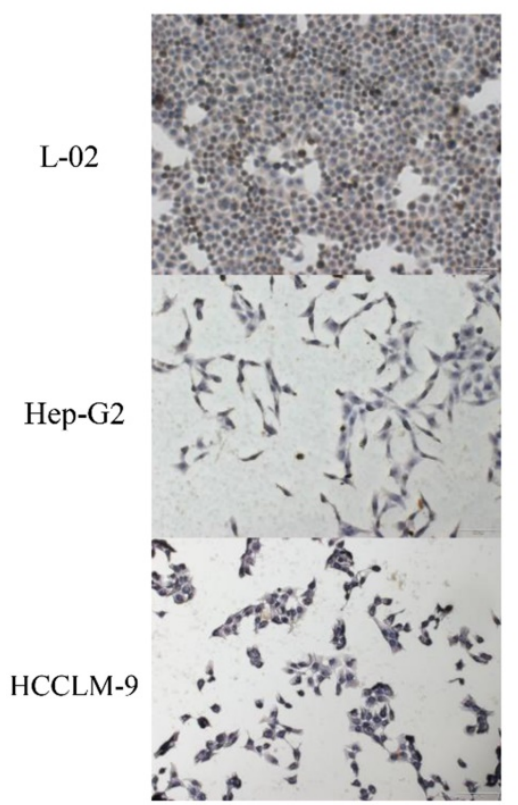

an important role in the development of HCC.

\section{Homer2 and Homer3 level in peripheral blood among subgroups}

We tested the expression of Homer2 and Homer3 in peripheral blood in 72 HCC patients, 59 cirrhosis patients, 52 hepatitis B, and 109 control cases.

Table 2 showed the main demographic and clinical characteristics of studied subjects. No difference was observed in risk factors including gender, age, smoking, alcoholism, whereas we found a significant difference in ALT, AST, TBIL, GGT and GLU among the groups. As illustrated in Figure 3, we found that Homer2 expression differed significantly between HCC and cirrhosis $(P<0.01)$, hepatitis $\mathrm{B}(P<0.01)$, and the controls $((P<0.01)$. For Homer3, its expression in HCC was lower than that in cirrhosis $(P<0.01)$, hepatitis B $(P<0.01)$ and the controls $(P<0.01)$ and Homer3 expression in cirrhosis $(P<$ $0.05)$ and hepatitis B $(P<$ 0.01 ) were lower than that in controls.

\section{Homer2 and Homer3 increased after operation in HCC}

We next explored whether peripheral blood expression level of Homer2 and Homer3 could be used to monitor tumor dynamics. 14 paired cases of HCC patients were enrolled to compare the Homer2 and Homer3 expression levels between preoperative and postoperative samples. We found that the Homer2 level increased in 9 of 14 HCC patients $(64.3 \%)$, and the Homer3 levels rose in 10 of 14 HCC patients $(71.4 \%)$, approximately 2 weeks after surgery (Figure 4).

Figure 1. Evaluation of Homer2 and Homer3 expression in $\mathrm{HCC}$ cell lines. Expression of Homer2 in HCC cell ines were lower than that in L-02 cell (A, C, E). Expression of Homer3 in HCC cell lines were lower than that in L-02 cells (B, D, F). $* P<0.05$. 
A

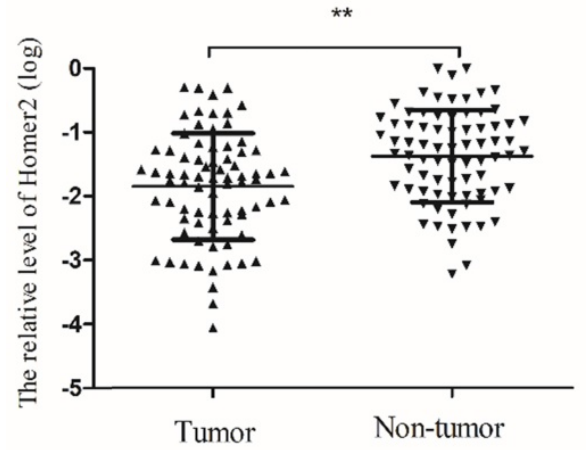

C

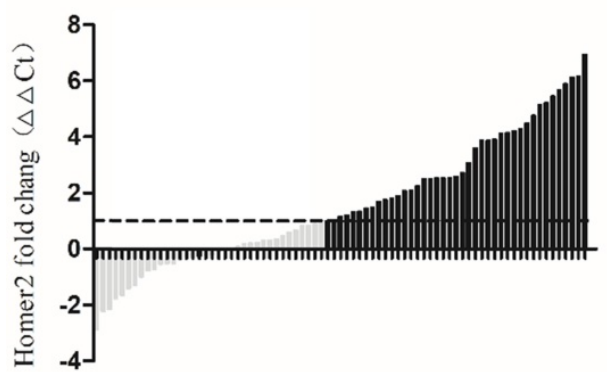

B

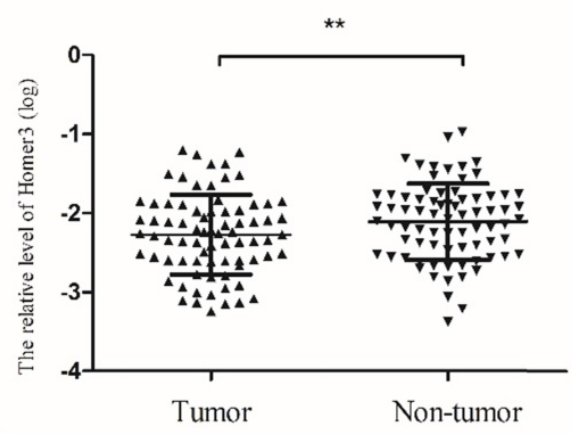

D

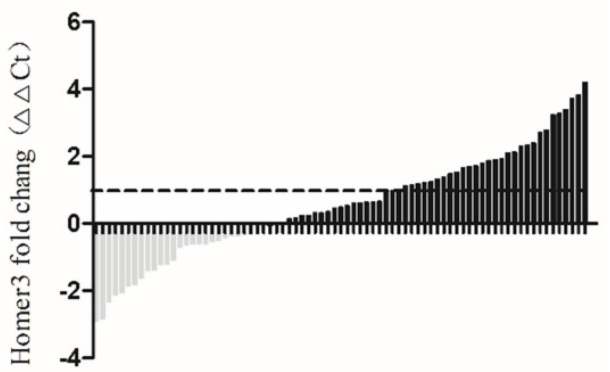

Figure 2. Homer2 and Homer3 expression in HCC tissues. The relative Homer2 and Homer3 expression were determined by RT-qPCR. $\triangle \mathrm{Ct}$ values were calculated by subtracting the GAPDH Ct value from the Homer2 or Homer3 $\mathrm{Ct}$ value. Smaller $\Delta \Delta \mathrm{Ct}$ value indicates higher expression. $\Delta \Delta \mathrm{Ct}=(\mathrm{Ct}$ Homer2/Homer3 $-\mathrm{Ct} \mathrm{GAPDH})$ of $\mathrm{HCC}-(\mathrm{Ct}$ Homer2/Homer3- Ct GAPDH) of NT]. (NT: paired noncancerous tissues of HCC). (A) Levels of Homer2 in tumor tissues are significantly lower than that in non-tumor tissues. (B) Levels of Homer3 in tumor tissues are significantly lower than that in non-tumor tissues. (C) Homer2 was reduced by at least twofold in $53.2 \%$ (41/77) of the HCC tissues. (D) Homer3 was downregulated in $62.3 \%(48 / 77)$ of $\mathrm{HCC}$ tissues. All data were analyzed using Student's t test. Data were presented as mean \pm SD, $* P<0.05$, $* * P<0.01$.

Table 1. Clinicopathological relevance analysis of Homer2 and Homer3 in HCC patients.

\begin{tabular}{|c|c|c|c|c|c|c|c|}
\hline \multirow[t]{2}{*}{ Characteristics } & \multirow[t]{2}{*}{$\mathbf{n}$} & \multicolumn{3}{|c|}{ Homer2 relative expression (log) } & \multicolumn{3}{|c|}{ Homer3 relative expression (log) } \\
\hline & & Mean \pm SD & $\mathbf{t}$ & $P$ & Mean \pm SD & $\mathbf{t}$ & $P$ \\
\hline Tissue & & & 6.072 & $0.0001^{* *}$ & & 3.011 & $0.004^{* *}$ \\
\hline $\mathrm{HCC}$ & 77 & $-1.85 \pm 0.83$ & & & $-2.27 \pm 0.62$ & & \\
\hline Adjacent Tissue & 77 & $-1.37 \pm 0.72$ & & & $-2.11 \pm 0.54$ & & \\
\hline Gender & & & -0.597 & 0.552 & & 0.583 & 0.562 \\
\hline Male & 71 & $-1.83 \pm 0.86$ & & & $-2.29 \pm 0.48$ & & \\
\hline Female & 6 & $-2.04 \pm 0.39$ & & & $-2.16 \pm 0.76$ & & \\
\hline Age & & & 1.419 & 0.160 & & -1.212 & 0.229 \\
\hline$<55$ & 39 & $-1.98 \pm 0.82$ & & & $-2.20 \pm 0.50$ & & \\
\hline$\geqq 55$ & 38 & $-1.71 \pm 0.83$ & & & $-2.34 \pm 0.50$ & & \\
\hline Alcoholism & & & 1.258 & 0.214 & & -0.031 & 0.975 \\
\hline Negative & 38 & $-1.89 \pm 0.83$ & & & $-2.31 \pm 0.49$ & & \\
\hline Positive & 18 & $-1.56 \pm 1.05$ & & & $-2.32 \pm 0.52$ & & \\
\hline Differentiation & & & -2.594 & $0.012^{*}$ & & 0.960 & 0.340 \\
\hline High/ Moderate & 58 & $-1.72 \pm 0.82$ & & & $-2.30 \pm 0.50$ & & \\
\hline Low & 9 & $-2.47 \pm 0.7$ & & & $-2.12 \pm 0.54$ & & \\
\hline Size & & & -0.332 & 0.741 & & -2.67 & $0.010^{* *}$ \\
\hline$<5 \mathrm{~cm}$ & 21 & $-1.75 \pm 0.84$ & & & $-2.04 \pm 0.47$ & & \\
\hline$\geqq 5 \mathrm{~cm}$ & 47 & $-1.82 \pm 0.86$ & & & $-2.38 \pm 0.49$ & & \\
\hline TNM stage & & & 0.001 & 0.999 & & -0.347 & 0.730 \\
\hline $\mathrm{I} \sim \mathrm{II}$ & 25 & $-1.75 \pm 0.7$ & & & $-2.3 \pm 0.57$ & & \\
\hline III $\sim$ IV & 29 & $-1.75 \pm 0.79$ & & & $2.35 \pm 0.46$ & & \\
\hline Tumor nodes & & & 0.447 & 0.657 & & 2.312 & $0.026^{*}$ \\
\hline Single & 31 & $-1.87 \pm 0.97$ & & & $2.44 \pm 0.44$ & & \\
\hline Multi & 8 & $-1.7 \pm 0.94$ & & & $-2.05 \pm 037$ & & \\
\hline Cirrhosis & & & 0.702 & 0.487 & & 0.987 & 0.330 \\
\hline Negative & 10 & $-2.08 \pm 1.22$ & & & $-2.34 \pm 0.59$ & & \\
\hline Positive & 29 & $-1.82 \pm 0.93$ & & & $-2.16 \pm 0.25$ & & \\
\hline $\operatorname{AFP}(n g / 1)$ & & & -1.509 & 0.136 & & 1.480 & 0.144 \\
\hline$<200$ & 33 & $-1.67 \pm 0.61$ & & & $-2.35 \pm 0.43$ & & \\
\hline$\geqq 200$ & 32 & $-1.98 \pm 0.99$ & & & $-2.17 \pm 0.54$ & & \\
\hline GGT (U/1) & & & 0.126 & 0.900 & & -2.618 & $0.001^{* *}$ \\
\hline$<55$ & 28 & $-1.81 \pm 0.66$ & & & $-2.10 \pm 0.45$ & & \\
\hline
\end{tabular}




\begin{tabular}{|c|c|c|c|c|c|c|c|}
\hline \multirow[t]{2}{*}{ Characteristics } & \multirow[t]{2}{*}{$\mathbf{n}$} & \multicolumn{3}{|c|}{ Homer2 relative expression (log) } & \multicolumn{3}{|c|}{ Homer3 relative expression (log) } \\
\hline & & Mean \pm SD & $\mathbf{t}$ & $P$ & Mean \pm SD & $\mathbf{t}$ & $P$ \\
\hline$\geqq 55$ & 42 & $-1.78 \pm 0.95$ & & & $-2.40 \pm 0.49$ & & \\
\hline $\mathrm{TP}(\mathrm{g} / \mathrm{l})$ & & & -2.195 & 0.032 * & & 0.950 & 0.345 \\
\hline$<65$ & 22 & $-1.51 \pm 0.64$ & & & $-2.37 \pm 0.51$ & & \\
\hline $65-85$ & 47 & $-1.96 \pm 0.87$ & & & $-2.24 \pm 0.49$ & & \\
\hline $\mathrm{Alb}(\mathrm{g} / \mathrm{l})$ & & & -0.520 & 0.605 & & -0.319 & 0.751 \\
\hline$<35$ & 12 & $-1.68 \pm 0.45$ & & & $-2.24 \pm 0.55$ & & \\
\hline $35-55$ & 57 & $-1.82 \pm 0.91$ & & & $-2.29 \pm 0.49$ & & \\
\hline
\end{tabular}

Data are shown as mean \pm standard deviation. Since we failed to collect all characteristics of the patients, the total number may not be 77 . TNM, tumor-node-metastasis. AFP, a-fetoprotein; GGT, $\gamma$-glutamyl transferase; TP, total protein; Alb, albumin. * $P<0.05,{ }^{* *} P \leq 0.01$.
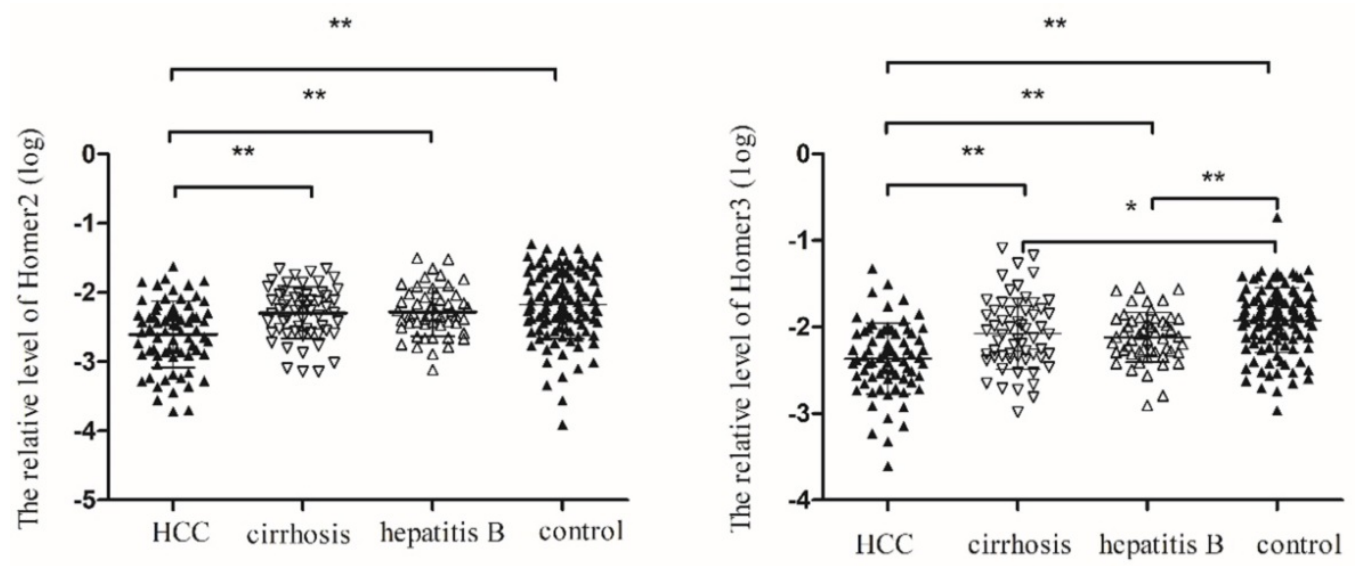

Figure 3. Homer2 and Homer3 expression in peripheral blood among subgroups. (A) Homer2 expression in HCC was lower than that in cirrhosis, hepatitis B and the controls. No differences were observed among other subgroups. (B) Homer3 expression in HCC was lower than that in cirrhosis, hepatitis B and the controls and Homer3 expression in cirrhosis and hepatitis B were lower than that in controls. No differences were observed between hepatitis B and cirrhosis. Data were analyzed using oneway ANOVA. $* P<$ $0.05, * * P<0.01$.

A

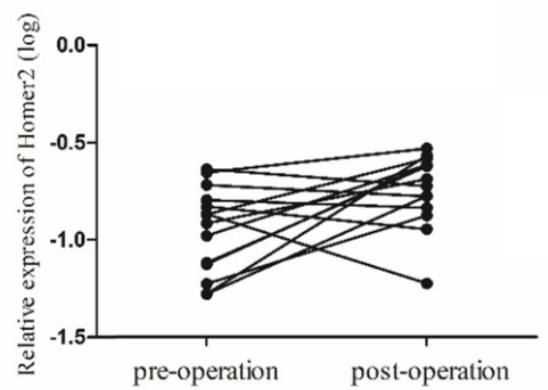

B

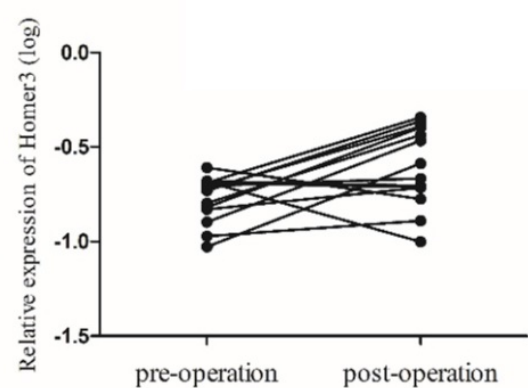

Figure 4. The expression levels of Homer2 and Homer3 in 14 paired preoperative and postoperative peripheral blood samples. (A) Levels of Homer2 was increased 2 weeks after surgical $(P=0.024)$; $(B)$ Levels of Homer 3 was increased 2 weeks after surgical $(P=0.022)$.

\section{Diagnostic value of Homer2 and Homer3}

To assess whether Homer 2 and Homer 3 could be used as potential diagnostic markers for HCC, ROC was carried out using 3 models: HCC vs Controls, HCC vs HBV, HCC vs cirrhosis (Table 3). Results showed that areas under the ROC curves of Homer2 and Homer3 to discriminate HCC patients from the controls was 0.743 and 0.798 respectively (Figure 5A). However, the diagnostic value of Homer2 and Homer3 for discrimination HCC from hepatitis B and cirrhosis was not obvious (Figure 5B-C). Combination of Homer2 and Homer3 possessed a moderate ability to discriminate HCC patients and controls with an area under the ROC curve of 0.809 (Figure 5D), while the area reached up to 0.900 when they were combined with AFP (Figure 5E).

\section{Discussion}

HCC is a public health burden due to its relatively high incidence and poor prognosis $[20,21]$. Studies have reported that over $50 \%$ of HCC cases are caused by persistent HBV infections [22]. To improve the prognosis of HBV-related HCC, it is of great importance to identify new appropriate biomarkers to diagnose HCC. Currently, AFP has been widely used for the diagnosis of HCC, but the results are far from satisfactory owing to the limited sensitivity and specificity $[23,24]$. Therefore, it is pivotal to explore new biomarkers to diagnose HCC at an early stage. 
Homer is well known as scaffold proteins [25] and plays an important role of in keeping the homestasis of calcium in intracellular [26]. Wu and his colleagues validated Homer1 was upregulated in intrahepatic cholangiocarcinoma than that in the adjacent tissues [27]. Our previous research showed that Homer1 could be a novel biomarker for HBV-HCC [17]. It's logic and interesting to study whether the other two family member Homer2 and Homer3 can be new biomarker for HCC.

Table 2. Characteristics of the studied participants.

\begin{tabular}{|c|c|c|c|c|c|}
\hline Characteristics & $\frac{\mathrm{HCC}}{\mathrm{N}=72}$ & $\begin{array}{l}\text { Cirrhosis } \\
N=59\end{array}$ & $\begin{array}{l}\text { Hepatitis B } \\
\mathrm{N}=52\end{array}$ & $\begin{array}{l}\text { Control } \\
\mathrm{N}=109\end{array}$ & $P$ \\
\hline Gender & & & & & $0.259 \mathrm{a}$ \\
\hline Male & 59 & 49 & 37 & 80 & \\
\hline Female & 13 & 10 & 15 & 29 & \\
\hline Age & & & & & $0.216^{\mathrm{a}}$ \\
\hline$<55$ & 26 & 27 & 16 & 54 & \\
\hline$\geqq 55$ & 46 & 32 & 36 & 65 & \\
\hline Smoking & & & & & $0.198 \mathrm{a}$ \\
\hline Negative & 38 & 39 & 27 & 70 & \\
\hline Positive & 34 & 20 & 25 & 39 & \\
\hline Alcoholism & & & & & $0.764^{\mathrm{a}}$ \\
\hline Negative & 45 & 38 & 35 & 76 & \\
\hline Positive & 27 & 21 & 17 & 33 & \\
\hline $\operatorname{ALT}(\mathrm{U} / 1)$ & $41(27,70)$ ** & $27(19,38)$ * & $79(31,283)$ ** & $20(17,27)$ & $<0.001^{\text {b }}$ \\
\hline AST (U/1) & $50(31,97)$ ** & $36(24,45) * *$ & $50(31,90)$ ** & $22(19,26)$ & $<0.001^{\mathrm{b}}$ \\
\hline TBIL $(\mu \mathrm{mol} / 1)$ & $21(14,27)$ ** & $22(14,36) * *$ & $21(13,79) * *$ & $13(11,16)$ & $0.002^{\mathrm{b}}$ \\
\hline GGT (U/1) & $147(61,296)^{\star * \star}$ & $40(22,104)^{*}$ & $54(20,109)^{* *}$ & $22(18,35)$ & $<0.001^{b}$ \\
\hline GLU (mmol/l) & $5.1(4.6,6.0)^{* *}$ & $4.6(4.2,5.9)$ & $4.6(4.2,5.4)$ & $4.4(4.7,5.3)$ & $0.002^{b}$ \\
\hline
\end{tabular}

Table 3. Comparisons of the AUC of the expression of Homer2 and Homer 3 for subgroups.

\begin{tabular}{|c|c|c|c|c|c|c|}
\hline Group & Gene & AUC & $95 \% \mathrm{CI}$ & $P$ & $\begin{array}{l}\text { Se } \\
(\%)\end{array}$ & $\begin{array}{l}S p \\
(\%)\end{array}$ \\
\hline HCC vs & Homer2 & 0.743 & $0.671-0.814$ & $<0.001$ & 61.5 & 77.8 \\
\hline Control & Homer3 & 0.798 & $0.730-0.863$ & $<0.001$ & 65.1 & 87.5 \\
\hline HCC vs & Homer2 & 0.696 & $0.605-0.789$ & $<0.001$ & 96.2 & 36.1 \\
\hline $\mathrm{HBV}$ & Homer3 & 0.698 & $0.606-0.790$ & $<0.001$ & 80.1 & 56.9 \\
\hline HCC vs & Homer2 & 0.691 & $0.601-0.780$ & $<0.001$ & 86.4 & 45.8 \\
\hline Cirrhosis & Homer3 & 0.691 & $0.600-0.782$ & $<0.001$ & 83.1 & 47.2 \\
\hline HCC vs & Homer2, Homer3 & 0.809 & $0.744-0.874$ & $<0.001$ & 72.5 & 83.3 \\
\hline Control $^{*}$ & Homer2, Homer3, AFP & 0.900 & $0.838-0.963$ & $<0.001$ & 83.3 & 83.9 \\
\hline
\end{tabular}

In our study, we revealed that Homer2 and Homer3 were downregulated in HCC tissues comparing with the adjacent noncancerous tissues. After analyzed the association between these two markers and clinical characteristics of HCC patients, we found that Homer2 expression was associated with tumor differentiation grade and TP level, while the expression of Homer3 was significantly related to tumor size, tumor nodes and GGT level. As we all know, an ideal clinical biomarker should be noninvasive and easily accessible. Thus, we subsequently tested the expression of Homer2 and Homer3 in peripheral blood leukocytes. In accordance with the results from tissue, the expression of Homer2 and Homer3 in HCC were both lower than that in healthy controls. By following up study, we found the increased expression level of both Homer2 and Homer3 after operation, indicating their potential value as surgical effects markers. At last, we identified that the combined AUC of Homer2, Homer3 and AFP reached up to 0.900 .

In our present study, we found the expression of both Homer2 and Homer3 were downregulated in HCC, which indicated a possible tumor suppressive role of Homer2 and Homer3 in HCC. In accordance with our study, Mhawech et al. [16] concluded that patients with higher Homer2 protein had a better outcome in endometrioid endometrial adenocarcinoma. However, other studies have validated that Homer might promote the process of tumorigenesis. Sun et al. [15] revealed that the expression of Homer2 was significantly correlated with the markedly reduced overall survival of RC patients. With evidence supporting a tumor suppressor role of Homer in some contexts but an oncogene in others. We therefore think that Homer may function differently in different types of malignancies.

It is well known that HBV infection causes a rapid immune response, causing a life-long immunity with acute self-limited infection in more than 95\% patients [28] and more than $50 \%$ of HCC are attributed to HBV infection [29]. Studies have validated that inappropriate cytotoxic $\mathrm{T}$ lymphocyte (CTL) response triggers cumulative hepatocyte damage to induce chronic inflammation and ultimately develop into HCC [30,31]. Huang et al. [18] found that Homer2 and Homer3 could negatively regulate the activation of T cell. Besides, Homer- 3 was found to inhibit the activation of serum response element (SRE) in T cells [32]. Thus, we deem that Homer2 and Homer3 may suppress the progress of HCC by inhibiting the activation of T cells. This may be a research point in our future studies.

In summary, Homer2 and Homer3 expression were downregulated both in HCC tissues and peripheral blood. The expression of Homer2 was associated with tumor differentiation grade and TP level, Homer3 was related to tumor size, tumor nodes and GGT level. In addition, the combination of Homer2, Homer3and AFP could differentiate HCC patients from healthy controls effectively. These findings firstly indicated that Homer2 and Homer3 might be useful makers to diagnose, even to monitor the surgery effect, Further studies in a large number of patients are required to confirm the usefulness of 
Homer2 and Homer3 in HCC.

A

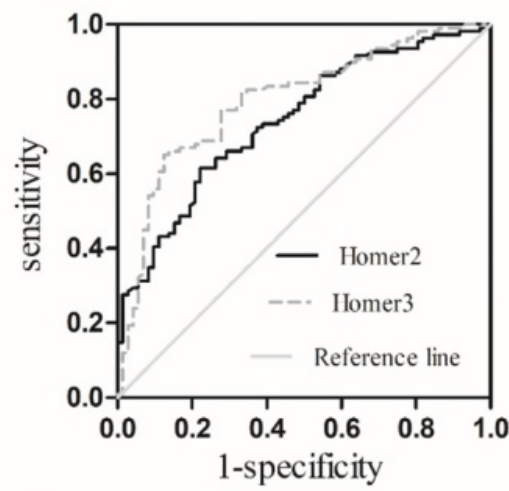

C
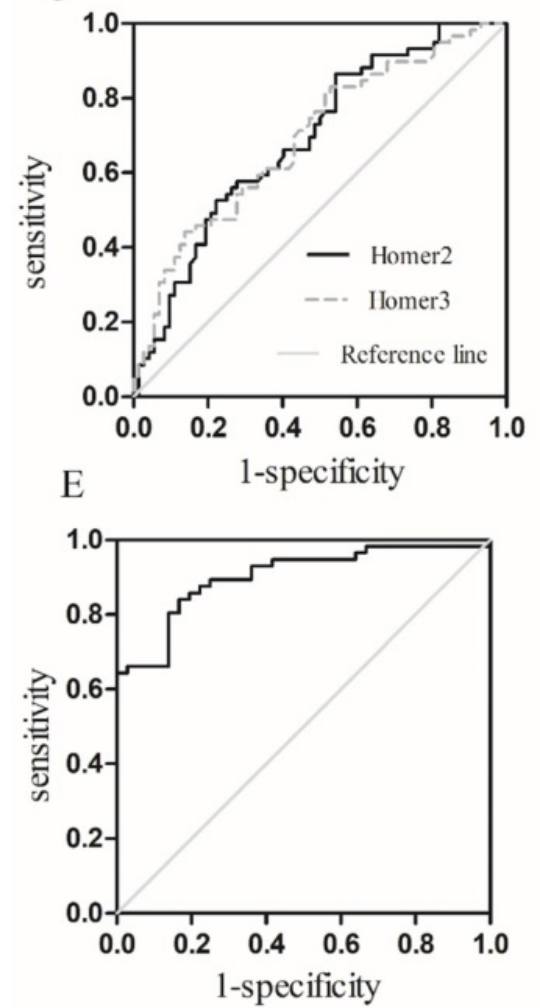

B

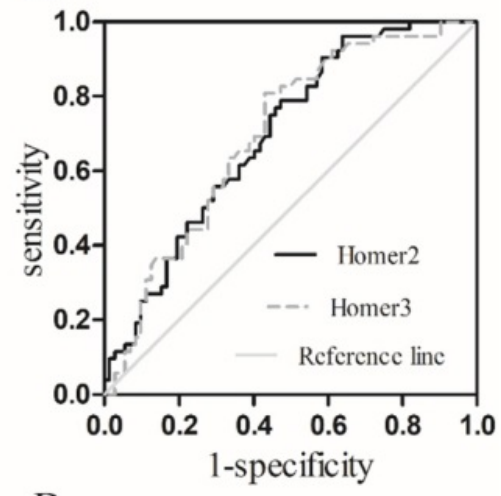

D

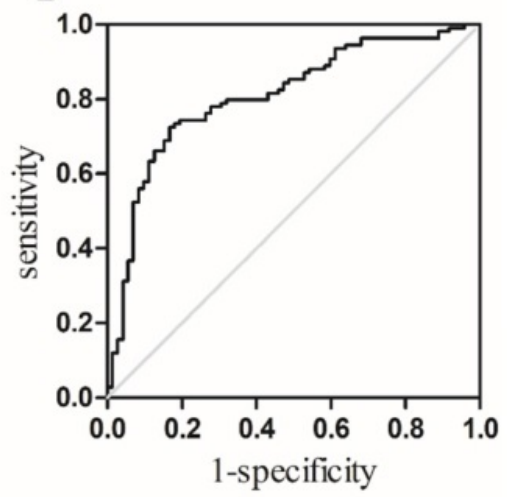

Figure 5. Receiver operating characteristic (ROC) curves. (A) Homer2 and Homer3 for HCC vs Controls; (B) Homer2 and Homer3 for HCC vs HBV; (C) Homer2 and Homer3 for HCC vs patients with cirrhosis; (D) the combination of Homer2 and Homer3 for HCC vs Controls; (E) the combination of Homer2, Homer3 and AFP for HCC vs Controls.

\section{Acknowledgments}

This study was supported by the Research of Homer ELISA Kit for Breast Cancer (201260523173-2), Science and Technology Innovation Cultivation Fund of Zhongnan Hospital of Wuhan University (znpy2018067) and National Basic Research Program of China (973 Program) (2012CB720605).

\section{Ethics approval and consent to participate}

Approved study protocol by the Medical Ethical Committee of Zhongnan Hospital of Wuhan University was got before the study was carried out and informed consent was obtained from all individuals involved in this study.

\section{Authors' contributions}

P.L. and CZ.L. were involved in review and analysis of the raw data, drafted the manuscript. J.W. , M.Z. and H.Z. contributed to clinical data collection. P.L. contributed to laboratory analysis. HY. C. and Paul F. Worley provided technical support. JC.T. contributed to the design of the study and revision of the manuscript. All authors read and approved the final manuscript. 


\section{Competing Interests}

The authors have declared that no competing interest exists.

\section{References}

1. Wu J, Yang S, Xu K, Ding C, Zhou Y, Fu X, et al. Patterns and Trends of Liver Cancer Incidence Rates in Eastern and Southeastern Asian Countries (1983-2007) and Predictions to 2030. Gastroenterology. 2018; 154: 1719-28.e5.

2. Shimada S, Mogushi K, Akiyama Y, Furuyama T, Watanabe S, Ogura T, et al. Comprehensive molecular and immunological characterization of hepatocellular carcinoma. EBioMedicine. 2019; 40: 457-70.

3. Teng L, Wang K, Liu Y, Ma Y, Chen W, Bi L. Based on Integrated Bioinformatics Analysis Identification of Biomarkers in Hepatocellular Carcinoma Patients from Different Regions. Biomed Res Int. 2019; 2019: 1742341.

4. Villanueva A Hepatocellular Carcinoma. Reply. N Engl J Med, 2019; 381: e2.

5. Makarova-Rusher OV, Altekruse SF, McNeel TS, Ulahannan S, Duffy AG, Graubard BI, et al. Population attributable fractions of risk factors for hepatocellular carcinoma in the United States. Cancer. 2016; 122: 1757-65.

6. Gu Y, Li X, Bi Y, Zheng Y, Wang J, Li X, et al. CCL14 is a prognostic biomarker and correlates with immune infiltrates in hepatocellular carcinoma. Aging (Albany NY). 2020; 12: 784-807.

7. Waghray A, Murali ARMenon KN Hepatocellular carcinoma: From diagnosis to treatment. World J Hepatol. 2015; 7: 1020-9.

8. Wei W, Liu M, Ning S, Wei J, Zhong J, Li J, et al. Diagnostic value of plasma HSP90a levels for detection of hepatocellular carcinoma. BMC Cancer. 2020; 20: 6 .

9. Gan W, Huang JL, Zhang MX, Fu YP, Yi Y, Jing CY, et al. New nomogram predicts the recurrence of hepatocellular carcinoma in patients with negative preoperative serum AFP subjected to curative resection. J Surg Oncol. 2018; 117: $1540-47$.

10. Shiraishi-Yamaguchi Y, Furuichi T. The Homer family proteins. Genome Biol. 2007; 8: 206.

11. Brakeman PR, Lanahan AA, O'Brien R, Roche K, Barnes CA, Huganir RL, et al. Homer: a protein that selectively binds metabotropic glutamate receptors. Nature. 1997; 386: 284-8.

12. Xiao B, Tu JC, Petralia RS, Yuan JP, Doan A, Breder CD, et al. Homer regulates the association of group 1 metabotropic glutamate receptors with multivalent complexes of homer-related, synaptic proteins. Neuron. 1998; 21: 707-16.

13. Stirewalt DL, Meshinchi S, Kopecky KJ, Fan W, Pogosova-Agadjanyan EL, Engel $\mathrm{JH}$, et al. Identification of genes with abnormal expression changes in acute myeloid leukemia. Genes Chromosomes Cancer. 2008; 47: 8-20.

14. Shen TY, Mei LL, Qiu YT, Shi ZZ. Identification of candidate target genes of genomic aberrations in esophageal squamous cell carcinoma. Oncol Lett. 2016; 12: 2956-61.

15. Sun M, Sun T, He Z, Xiong B. Identification of two novel biomarkers of rectal carcinoma progression and prognosis via co-expression network analysis. Oncotarget. 2017; 8: 69594-609.

16. Mhawech-Fauceglia P, Walia S, Yessaian A, Machida H, Matsuo K, Lawrenson K. Overexpression of HOMER2 predicts better outcome in low-grade endometrioid endometrial adenocarcinoma. Pathology. 2018; 50: 499-503.

17. Luo $\mathrm{P}$, Feng $\mathrm{X}$, Jing $\mathrm{W}$, Zhu M, Li N, Zhou H, et al. Clinical and Diagnostic Significance of Homer1 in hepatitis B virus-induced Hepatocellular Carcinoma. J Cancer. 2018; 9: 683-89.

18. Huang GN, Huso DL, Bouyain S, Tu J, McCorkell KA, May MJ, et al. NFAT binding and regulation of $\mathrm{T}$ cell activation by the cytoplasmic scaffolding Homer proteins. Science. 2008; 319: 476-81.

19. Gabrielson A, Wu Y, Wang H, Jiang J, Kallakury B, Gatalica Z, et al. Intratumoral CD3 and CD8 T-cell Densities Associated with Relapse-Free Survival in HCC. Cancer Immunol Res. 2016; 4: 419-30.

20. Zahid KR, Han S, Zhou F, Raza U. Novel tumor suppressor SPRYD4 inhibits tumor progression in hepatocellular carcinoma by inducing apoptotic cell death. Cell Oncol (Dordr). 2019; 42: 55-66.

21. Karaosmanoğlu O, Banerjee S, Sivas H. Identification of biomarkers associated with partial epithelial to mesenchymal transition in the secretome of slug over-expressing hepatocellular carcinoma cells. Cell Oncol (Dordr). 2018; 41: $439-53$.

22. Levrero MZucman-Rossi J. Mechanisms of HBV-induced hepatocellular carcinoma. J Hepatol. 2016; 64: S84-s101.

23. Farinati F, Marino D, De Giorgio M, Baldan A, Cantarini M, Cursaro C, et al. Diagnostic and prognostic role of alpha-fetoprotein in hepatocellular carcinoma: both or neither? Am J Gastroenterol. 2006; 101: 524-32.

24. Jang ES, Jeong SH, Kim JW, Choi YS, Leissner PBrechot C. Diagnostic Performance of Alpha-Fetoprotein, Protein Induced by Vitamin K Absence, Osteopontin, Dickkopf-1 and Its Combinations for Hepatocellular Carcinoma. PLoS One. 2016; 11: e0151069.

25. Tu JC, Xiao B, Yuan JP, Lanahan AA, Leoffert K, Li M, et al. Homer binds a novel proline-rich motif and links group 1 metabotropic glutamate receptors with IP3 receptors. Neuron. 1998; 21: 717-26.

26. Pouliquin P, Dulhunty AF. Homer and the ryanodine receptor. Eur Biophys J. 2009; 39: 91-102.
27. Wu SY, Yu MX, Li XG, Xu SF, Shen J, Sun Z, et al. Identification of Homer1 as a potential prognostic marker for intrahepatic cholangiocarcinoma. Asian Pac J Cancer Prev. 2014; 15: 3299-304.

28. Rehermann B, Thimme R. Insights From Antiviral Therapy Into Immune Responses to Hepatitis B and C Virus Infection. Gastroenterology. 2019; 156: 369-83.

29. Hao X, Chen Y, Bai L, Wei H, Sun R, Tian Z. HBsAg-specific CD8(+) T cells as an indispensable trigger to induce murine hepatocellular carcinoma. Cell Mol Immunol. 2021; 18: 128-37.

30. Bertoletti A, Kennedy PTF, Durantel D. HBV infection and HCC: the 'dangerous liaisons'. Gut. 2018; 67: 787-88.

31. Lim CJ, Lee YH, Pan L, Lai L, Chua C, Wasser M, et al. Multidimensional analyses reveal distinct immune microenvironment in hepatitis $\mathrm{B}$ virus-related hepatocellular carcinoma. Gut. 2019; 68: 916-27.

32. Ishiguro $\mathrm{K}$, Xavier R. Homer-3 regulates activation of serum response element in T cells via its EVH1 domain. Blood. 2004; 103: 2248-56. 